\title{
Stochastic Modeling on Drug Efficacy in Self Drug Administration Health Problems
}

\author{
P. Tirupathi Rao' ${ }^{1}$, M. Venkateswaran ${ }^{1}$, R. Vishnu Vardhan ${ }^{2}$ \\ ${ }^{1}$ Department of Statistics, S. V. University, Tirupati, India; ${ }^{2}$ Department of Statistics, Pondicherry University, Puducherry, India. \\ E-mail: \{drtrpadi, venky.swaran, rvvcrr\}@gmail.com \\ Received July $12^{\text {th }}, 2011$; revised September $3^{\text {rd }}, 2011$; accepted October $9^{\text {th }}, 2011$.
}

\begin{abstract}
Evaluation of drug impact both on positive and negative sides is very important to monitor the drug administration during the treatment. The effectiveness of drug is a variable due to various chance and assignable causes. Evaluation of drug efficacy through conventional mathematical modeling is the existing practice. In this paper we proposed a Stochastic model for measuring the drug efficacy for Non-Clinical and short term drug administration practices. Sensitivity analysis is carried out to observe the model behavior. Development of computer software and desktop templates to this model will provide effective decision support systems for health caretakers.
\end{abstract}

Keywords: Stochastic Modeling, Drug Efficacy, Self Drug Administration

\section{Introduction}

Treatment with drugs is one of the customary methods to cure diseases. Drug administration on the disease control in under developed and developing countries is mostly on non-clinical environment, particularly for the short term and seasonal related disease treatments like cold, fever, influenza, cough etc. Major category/population of the patients in India are getting the treatment through non competent medical supervision. It leads to random picking of drug at the choice of either individual own behalf or Medical Shop holders or Registered Medical Practitioners etc. This sort of situation provokes non-assurance of drug effectiveness on the control of the disease. Decision making on drug picking has to be made on various parameters of drug administration like quantity of drug per unit time, number of times (frequency) of drug administration per unit time period and many other similar factors. The health care taking must be supported by competent screening procedures with proper health indicators on the efficacy of drug under usage. Drug dosage level less than the required and more than the sufficient are unwanted as the farmer leads to the body drug resistant and the later may cause hazards to general health of the patient. Therefore the limits on size of the drug dose are playing vital role in regulations of disease intensity.

Random and erratic usage of drug without scientific approach will make drug administration more vulnerable. It always act as double edged weapon through either wanted or unwanted impact. Spontaneous selection of drug based on purely chance manner requires due attention of the researchers. Assessment on the levels of drug effecttiveness on the targeted disease control is the need of the hour. Measuring the levels of both positive and negative effectiveness is possible through the construction of a relevant Mathematical model. Our study can measure the net effectiveness of drug by considering the linear combination of positive and negative influences of drug on the disease control. This study will assist as the health management tool for decision support system. It can assess the influence of drug either of proper usage or of abuse by obtaining various Statistical measures of lower and higher order.

A suitable formulation of the bio-systems into mathematical formulation and transforming classic mathematiccal environment into statistical/empirical situations is pivotal. There is much literature evidence on modeling the drug efficiency in Deterministic environment. The work so far carried out is mostly concentrated on drug administration for long term treatments. A few work is reported in the literature on measuring the drug effectiveness on the treatment of short term duration.

Drug administration on clinical environment can be done in several different methods [1]. The model of drug resistance from gene amplifications could be studied through the policies of optimal control [2]. Several asymptotic properties of infinite dimensional model drug resis- 
tance evolutions are available [3]. The phamalogemmics can be studied through drug disposition, drug targets and its side effects [4]. The Legand efficiency indices are considered as the guide spots in the discovery of drug effecttiveness [5]. Drug efficiency indices were also constructed with structured based calculation [6]. The evaluation of drug efficacy for long term Treatment problems can be done through stochastic models. The environment of drug administration with ' $r$ ' alternatives and each alternative is of completely random is considered. The treatment is administered for $\mathrm{N}$ days for which the effectiveness of drug has varying chances. The selected drug for treating the disease is correct with certainty for $N_{1}$ days, the choice of correct selection is equal to $1 / k$ for $N_{k}$ days where $k=1,2, \cdots, r$. Further the total period of treatment is $N=\sum_{i=1}^{k} N_{i}$. The model has applied to study the drug effectiveness for long term treatments like cancer, T.B. and skin related diseases [7]. In this paper we have developed a stochastic model for evaluating the effecttiveness of drug for the treatments of short durations. This study has focused the attention on calculating the drug efficacy when the choice of the drug is random and having positive and negative impacts. The net effectiveness of drug is a linear combination of both positive and negative impacts with some weight coefficients.

\section{Stochastic Model and Statistical Measures}

In this study we develop a stochastic model for measureing the effectiveness of the drug by considering the following assumptions based on the problems of the patients; Problems of Drug selection and Problems of Drug administration.

- The patients may get ill health such as cold, seasonal fever, headaches and similar non-chronic and short term diseases at a random time.

- The patient is not having required knowledge on the drug administration parameters such as (i) The dosage level of drug; (ii) The frequency of drug administration per unit time (iii) time between two spells of drug administration; (iv) neither the patient nor healthcare taker on his behalf is aware on the side effects of the drug.

- The patients may pick the drug either on his own choice or from the suggestion of a medical shopkeeper or from other similar people.

- He/She may initiate the usage of drugs with a group of medicines initially but some medicines may be missed during the administration period.

- There is every likely to skip some spells of taking medicines during the course period of drug administration.

- The drug usage may be stopped abruptly at any point of time on various reasons like he/she may get relief from the problem, they may not enough stock of drug to use for the required course period.

- Patient will select the drug of option among available drugs.

- The usage of medicines may be either a single drug or a group of drugs.

- $\mathrm{He} / \mathrm{She}$ administered the drug one or more pills for one time and more than one time in a day and more than one day in over all administration.

This model can measure the drug effectiveness for intermediate evaluation during treatment period. Due to various unexplained reasons and the varying levels of health conditions of the patient during the course period, the effectiveness of drug has to be considered as random variable and it is obtained as measure of efficiency (e). It is the ratio of the output achieved to the input used.

$$
\mathrm{e}=\frac{\text { Acheived Ouput }}{\text { Used Inputs }}
$$

Output can be obtained through various Statistical means and parametric observations with pre and post-drug administration datasets.

The model is constructed to obtain stochastic processes so as the behavior of a random variable $Z$ can be analyzed. It is the Net effectiveness of drug, expressed as $Z=a X+b Y$; where $a, b$ are the weight age coefficients corresponding to $X, Y$ respectively. They are obtained through preparation of frequency tables and pattern identifications in the data.

$\mathrm{X}$ is a Bernoulli variable assuming values 1,0 for the drug impact when it has positive effect (1) and drug non positive effect (0). "Positive effectiveness of drug" does implies that the drug has perform its task on healing the problem, what exactly it is meant for. "Non positive effectiveness of drug" does implies that the task of drug assignment may be un served as a neutral impact on overall effectiveness in healing the problem.

$\mathrm{Y}$ is another Bernoulli variable assuming values 1, 0 for the drug impact has negative effect (1) and the drug has non-negative effect (0). "Negative effectiveness of the drug" does implies that usage of drug may cause some loss to the general health due to side effects in mismatching of drug that is consumed and the health problem that is under study. "Non-negative effectiveness of the drug" does mean that usage of drug may not harm the overall health of the patient or there is a neutral negative effectiveness.

Let $p_{1}$ be the probability of positive impact and $p_{2}$ be the probability of non-positive impact. The probability distribution for the same is $P(X=i)=p_{1}$; for $I=1 ; P(X$ $=i)=p_{0}$; for $I=0$; This probability distribution is obtained from the stochastic process of an indicator variable $\left\{X_{i} ; I=0,1\right\}$; further $p_{1}, p_{0} \geq 0 ; p_{1}+p_{0}=1$. The $r^{\text {th }}$ 
order raw moment for the above probability distribution is $\mu_{r}^{\prime}(X)=p_{1}$.

Similarly, let $\mathrm{q}_{1}$ be the probability of Negative impact and $\mathrm{q}_{2}$ be the probability of non negative impact. The stochastic process of $Y$ is $\left\{Y_{j} ; j=0,1\right\}$ and the probability distribution is

$P(Y=j)=q_{1}$; for $j=1 ; P(Y=j)=q_{0} ;$ for $j=0$; and $q_{1}+$ $q_{0}=1 . q_{1}, q_{0} \geq 0$ the $k^{\text {th }}$ order raw moments of the distribution is $\mu_{k}^{\prime}(Y)=q_{1}$.

The random variables $X$ and $Y$ are assumed to be independent as the occurrence of events say positive and negative effective nesses are no way influenced by one with the other so that bivariate stochastic process is $\left\{\left(X_{i}\right.\right.$, $\left.\left.Y_{j}\right) ; i, j=0,1\right\}$ and its probability function is $\mathrm{P}(X=i, Y=\mathrm{j})$ $=P(X=\mathrm{i}) . \mathrm{P}(Y=j)$ for $i, j=(0,1)$ and the $r^{\text {th }}$ order raw moment of joint probability distribution is $\mu_{r}^{\prime}(X, Y)=p_{1} q_{1}$.

$Z$ as combined random variable can measure the net drug effectiveness by considering both positive and negative impacts together. Usually ' $b$ ' has a negative coefficient so as $Z$ value become $Z=a X-b Y$ the study has to be focused on $Z$ variable as a mixture of $X$ and $Y$ probability distribution. The Stochastic Process of $Z$ is $=$ $\left\{Z_{k}, k=0,1\right\}$ where $Z_{i, j}=a X_{i}-b Y_{j}$ the probability distribution is

$$
\begin{aligned}
P(Z) & =a\left(\begin{array}{l}
1 \\
x
\end{array}\right)\left(p_{1}\right)\left(p_{0}\right)^{(1-x)}-b\left(\begin{array}{l}
1 \\
y
\end{array}\right)\left(q_{1}\right)^{y}\left(q_{0}\right)^{(1-y)} ; \\
x, y & =0,1=0 \text { Otherwise. }
\end{aligned}
$$

For all practical purposes we may consider $b$ as $(-1)$ (b). The Statistical measures are obtained using relation $Z$ $=a X+b Y$ and $b=(-1) b$ thus the results are:

1) The $r^{\text {th }}$ order origin (raw) moment of $Z$ is:

$$
\mu_{r}^{\prime}(Z)=\sum_{k=0}^{r}(-1)^{k}\left(\begin{array}{l}
r \\
k
\end{array}\right)\left(a p_{1}\right)^{r}\left(b q_{1}\right)^{k} .
$$

By assuming $r=1,2,3$, 4, we can obtain the first four raw moments. With the help of these four, we can get Mean, S. D., Variance, C. V. Coefficients of Skewness and Kurtosis etc.

2) Average or Mean Effectiveness of Drug is: $=a \cdot p_{1}-b \cdot q_{1}$.

3) Variability of Drug Effectiveness is: $=a^{2} \cdot p_{1} \cdot p_{0}+b^{2} \cdot q_{1} \cdot q_{0}$.

4) Standard Deviation $=\left(a^{2} p_{1} p_{0}+b^{2} q_{1} q_{0}\right)^{\frac{1}{2}}$.

5) Coefficient of Variation of Drug Effectiveness

$$
=\frac{\left[a^{2} \cdot p_{1} \cdot p_{0}+b^{2} \cdot q_{1} \cdot q_{0}\right]^{\frac{1}{2}}}{a \cdot p_{1}-b \cdot q_{1}} \text {. }
$$

6) The $3^{\text {rd }}$ Central Moment is:

$$
\begin{aligned}
\mu_{3}= & a^{3} \cdot p_{1} \cdot\left(1-3 \cdot p_{1}+2 \cdot p_{1}^{2}\right) \\
& -b^{3} \cdot q_{1} \cdot\left(1-3 \cdot q_{1}+2 \cdot q_{1}^{2}\right)
\end{aligned}
$$

7) The $4^{\text {th }}$ Central Moment is:

$$
\begin{aligned}
\mu_{4}= & a^{4} \cdot p_{1} \cdot\left(1-4 \cdot p_{1}+6 \cdot p_{1}^{2}-3 \cdot p_{1}^{3}\right) \\
& +b^{4} \cdot q_{1}\left(1-4 \cdot q_{1}+6 \cdot q_{1}^{2}-3 \cdot q_{1}^{3}\right) \\
& +6 \cdot a^{2} \cdot b^{2} \cdot p_{1} \cdot q_{1}\left(p_{0}-q_{1}+p_{1} \cdot q_{1}\right)
\end{aligned}
$$

8) The coefficient of Skewness is:

$$
\left(\beta_{1}\right)=\frac{\left[a^{3} \cdot p_{1} \cdot\left(1-3 \cdot p_{1}+2 \cdot p_{1}^{2}\right)-b^{3} \cdot q_{1}\left(1-3 \cdot q_{1}+2 \cdot q_{1}^{2}\right)\right]^{2}}{\left(a^{2} \cdot p_{1} \cdot p_{0}+b^{2} \cdot q_{1} \cdot q_{0}\right)^{3}}
$$

9) The coefficient of Kurtosis is: (see Equation (9))

10) The Moment generating function of $Z$ is:

$$
M_{Z}(t)=\sum_{m=0}^{1} \sum_{n=0}^{1} p_{m} q_{n} e^{(m a-n b) t}
$$

11) Characteristic function of $Z$ is:

$$
\phi_{z}(t)=\sum_{m=0}^{1} \sum_{n=0}^{1} p_{m} q_{n} e^{(m a-n b) i t} ; \text { for } i^{2}=1
$$

12) Probability generating function of $Z$

$$
P_{z}(S)=\sum_{m=0}^{1} \sum_{n=0}^{1} p_{m} q_{n} S^{(m a-n b)}
$$

\section{Methodology}

The following methodology is considered for measuring the drug efficacy. The usual practices that are happened in clinical treatments are based on the readings of pre and post tests. In general the diagnosis procedures at clinical treatment are based on the screening tests. If we consider an example of screening tests of fever, the intensity of fever can be assessed with Temperature of the body, Pulse rate of the nerve, no of breathes of the patient, skin temperature, rectal temperature, etc.

Fever may be caused due to so many reasons like infections, inflammation, indigestion, insect bite, etc and many unexplained also. Let us assume that a patient is getting treatment for a fever. He has no idea about the reason for getting fever. His objective is to get rid of fever by consuming some pills. In this context the drug may give the effect on four fold namely:

$$
\beta_{2}=\frac{a^{4} \cdot p_{1}\left(1-4 \cdot p_{1}+6 \cdot p_{1}^{2}-3 \cdot p_{1}^{3}\right)+b^{4} \cdot q_{1}\left(1-4 \cdot q_{1}+6 \cdot q_{1}^{2}-3 \cdot q_{1}^{3}\right)+6 \cdot a^{2} \cdot b^{2} \cdot p_{1} \cdot q_{1}\left(p_{0}-q_{1}+p_{1} \cdot q_{1}\right)}{\left(a^{2} \cdot p_{1} \cdot p_{0}+b^{2} \cdot q_{1} \cdot q_{0}\right)^{2}}
$$


1) Positive effect: The drug shall decrease the temperature through the means of suppressing the reasons of causing fever.

2) Non-Positive effect: The drug may not decrease the temperature as it has no influence on suppression of the fever causing factors.

3) Negative effect: The drug may give adverse effects on general health of the patient causing unwanted side effects.

4) Non-Negative effect: The drug may not give unwanted side effects irrespective of its positive or non-positive effects.

The concepts of Non-Positive and Non-negative effects of drug, though they appears to be same, we have considered those two are significantly differed as the impact factor of non-positive effect of the drug is not equal to the impact factor of non-negative effect of drug.

The coefficient of positive effectiveness (a), may obtained as influenced relation of many factors. It may otherwise defined as $a=\bar{e}_{p}=\frac{\sum_{i=1}^{r} e_{p i}}{r} ; e_{p i}$ is the Positive efficiency measure of $i^{\text {th }}$ factor; $i=1,2, \cdots, r$; As the usual parameters of a disease like fevers are Body temperature, Heartbeat/Pulse rate, number of breathes, skin temperature etc.

Let us consider the first parameter namely temperature,

$e_{p_{1}}=\left|\frac{\left(\begin{array}{c}\text { initial temperature } \\ \text { before using drug }\end{array}\right)-\left(\begin{array}{c}\text { Temperature } \\ \text { after using drug }\end{array}\right)}{\text { Quantity of drug used }}\right|=\frac{t b-t a}{q}$.

[Example: If $\mathrm{tb}=103^{\circ} ; \mathrm{ta}=102^{\circ} ; \mathrm{q}=500 \mathrm{mg}$ then

$\left.e_{p_{1}}=\frac{103-102}{500}=\frac{1}{500}=\frac{0.2}{100}=0.002\right]$.

Let Heart beat count is the $2^{\text {nd }}$ parameter, then

$$
\begin{aligned}
e_{p_{2}} & =\left|\frac{\left(\begin{array}{c}
\text { initial heartbeat } \\
\text { before using drug }
\end{array}\right)-\left(\begin{array}{l}
\text { Heartbeat count } \\
\text { after using drug }
\end{array}\right)}{\text { quantity of drug used }}\right| . \\
& =\left|\frac{\mathrm{HB}(\text { pre })-\mathrm{HB}(\text { post })}{q}\right|
\end{aligned}
$$

If number of breathes per unit time is the $3^{\text {rd }}$ parameter then

$$
e_{p_{3}}=\left|\frac{\mathrm{HB}(\text { pre })-\mathrm{HB}(\text { post })}{\text { quantity of drug used }}\right| .
$$

In general sense, $e_{p_{i}}=\left|\frac{\left(\begin{array}{c}\text { previous reading } \\ i^{\text {th }} \text { parameter }\end{array}\right)-\left(\begin{array}{c}\text { post reading } \\ i^{\text {th }} \text { parameter }\end{array}\right)}{\text { used drug quantity }}\right|$

$e_{p_{i}}=\left|\frac{(\text { reading of pre test }(i))-(\text { reading of post test }(i))}{\mathrm{q}}\right|$

Combining all the above, $a=\bar{e}_{p}=\sum_{i=1}^{r} \frac{e_{p i}}{r}$, where ' $r$ ' is the total number of factors on which the positive effecttiveness of drug is attained. Similarly the coefficient of Negative effectiveness (b) may also be influenced by non suitability or mismatching of drug to the disease under treatment.

For example: usage of certain drug leads to increased loss of albumin through urine.

$$
\begin{aligned}
e l_{1} & =\left|\frac{\left(\begin{array}{c}
\text { Albumni loss } \\
\text { before drug use }
\end{array}\right)-\left(\begin{array}{c}
\text { Albumni loss } \\
\text { after drug use }
\end{array}\right)}{\text { quantity of drug }}\right|, \\
& =\left|\frac{\text { Alb }(\text { pre test })-\text { Alb (post test })}{q}\right|
\end{aligned}
$$

$e l_{1}$ is the total negative effectiveness of drug. If there are $\mathrm{k}$ types of factors that are making the drug negative effectiveness. Then the overall negative effectiveness is

obtained as $b=\bar{e}_{l}=\sum_{j=1}^{r} \frac{e_{l_{j}}}{r}$; The coefficients of both positive effectiveness and negative effectiveness are influenced by all the relevant factors. This measure is statistically valid as the readings are considered on various parameters of the problem under study.

The chances of positive effectiveness ' $p_{1}$ ', Non-positive effectiveness ' $p_{0}$ ' are obtained as the proportions of their impact on overall usage. These values are usually obtained from the relative frequency distributions or from some historical data. These observations may also obtained from the pharmacology experimental studies. Let a drug is applied on ' $m$ ' living beings and of which ' $x$ ' are having the positive result and the rest $(m-x)$ are not having the positive effectiveness. Then $p_{1}=\frac{x}{m}$ and $p_{0}=1-p_{1}=1-\frac{x}{m}=\frac{m-x}{m}$.

The chances of negative impact can also be studied on similar lines. Let a drug is applied on ' $n$ ' living beings of which ' $y$ ' are having the negative impact and the remaining $n-y$ are not having negative results then the chances are 
$q_{1}=\frac{y}{n}$ and $q_{0}=1-q_{1}=1-\frac{y}{n}=\frac{n-x}{n}$.

\section{Numerical Illustration and Sensitivity Analysis}

In order to get the insights of the drug efficiency, a data is considered with inputs $p_{1}, q_{1}, a$ and $b$. The outputs like average drug effectiveness, variability in the drug effecttiveness, coefficient of variation, coefficient of Skewness, coefficient of kurtosis, etc are calculated with software MATHCAD. The numerical data is placed in Tables 1, mean response and the variability of the drug impact are analyzed. It is observed that Average drug efficiency is an increasing function of $p_{1}$, and it is negative when $p_{1}<$ $q_{1}, a<b$ where as Mean efficiency is an increasing function of $p_{1}$, and it is positive when $p_{1}>q_{1}, a<b$ when other parameters are constants. It is further observed that Average drug efficiency is an increasing function of $p_{1}$, and it is negative when $p_{1}<q_{1}, a>b$ where as Mean efficiency is an increasing function of $p_{1}$, and it is positive when $p_{1}>q_{1}, a>b$ when other parameters are constants. It is observed that Average drug efficiency is a decreasing function of $q_{1}$, when $p_{1}>q_{1}, a<b$ where as Mean efficiency is a decreasing function of $q_{1}$, and it is negative when $p_{1}<q_{1}, a<b$ when other parameters are constants. It is further observed that Average drug efficiency is a decreasing function of $q_{1}$, and it is positive when $p_{1}>q_{1}$, $a>b$ where as Mean efficiency is a decreasing function of $q_{1}$, and it is positive when $p_{1}<q_{1}, a>b$ when other parameters are constants.

It is observed from Figure 1 that Average drug efficiency is an increasing function of $p_{1}$, and it is negative when $p_{1}<q_{1}, a<b$, where as Mean efficiency is an increasing function of $p_{1}$ and it is positive when $p_{1}>q_{1}, a$ $<b$ when other parameters are constants. It is further observed that Average drug efficiency is an increasing function of $p_{1}$, and it is negative when $p_{1}<q_{1}, a>b$ where as Mean efficiency is an increasing function of $p_{1}$, and it is positive when $p_{1}>q_{1}, a>b$ when other parameters are constants. It is observed that Average drug efficiency is a decreasing function of $q_{1}$, when $p_{1}>q_{1}, a<b$ where as Mean efficiency is a decreasing function of $q_{1}$, and it is negative when $p_{1}<q_{1}, a<b$ when other parameters are constants. It is further observed that Average drug efficiency is a decreasing function of $q_{1}$, and it is positive when $p_{1}>q_{1}, a>b$ where as Mean efficiency is a decreasing function of $q_{1}$, and it is positive when $p_{1}<q_{1}$, $a>b$ when other parameters are constants.

It is observed that the variability of drug efficiency is an increasing function of $p_{1}$ when $p_{1}<q_{1}, a<b$; it is decreasing function of $p_{1}$ when $p_{1}>q_{1}, a<b$; Further it is observed that the variability of drug efficiency is a decreasing function of $p_{1}$ when $p_{1}<q_{1}, a>b$; it is decreas- ing function of $p_{1}$ when $p_{1}>q_{1}, a>b$ when other parameters are constants. It is observed that the variability of drug efficiency is an increasing function of $q_{1}$ when $p_{1}$ $>q_{1}, a<b$; it is decreasing function of $q_{1}$ when $p_{1}<q_{1}, a$ $<b$; Further it is observed that the variability of drug efficiency is an increasing function of $q_{1}$ when $p_{1}<q_{1}, a$ $>b$; it is a decreasing function of $q_{1}$ when $p_{1}<q_{1}, a>b$ when other parameters are constants.

It is observed that Average drug efficiency is an increasing function of $a$, it is negative when $p_{1}<q_{1}, a<b$; whereas Mean efficiency is an increasing function of $a$, and it is positive when $p_{1}<q_{1}, a>b$ when other parameters are constants. It is further observed that Average drug efficiency is an increasing function of $a$, and it is negative when $p_{1}>q_{1}, a<b$ where as Mean efficiency is an increasing function of $a$, and it is positive when $p_{1}>q_{1}$, $a>b$ when other parameters are constants. It is observed that Average drug efficiency is a decreasing function of $b$, it is negative when $p_{1}<q_{1}, a<b$; whereas Mean efficiency is a decreasing function of $b$, and it is positive when $p_{1}<q_{1}, a<b$ when other parameters are constants. It is further observed that Average drug efficiency is a decreasing function of $b$, and it is negative when $p_{1}>q_{1}$, $a<b$ where as Mean efficiency is a decreasing function of $b$, and it is positive when $p_{1}>q_{1}, a>b$ when other parameters are constants.

It is observed that the variability of drug efficiency is an increasing function of $a$ when $p_{1}<q_{1}, a<b$; it is an increasing function of $a$ when $p_{1}<q_{1}, a>b$; Further it is observed that the variability of drug efficiency is an increasing function of $a$ when $p_{1}>q_{1}, a<b$; it is an increasing function of $a$ when $p_{1}>q_{1}, a>b$ when other parameters are constants. It is observed that the variability of drug efficiency is an increasing function of $b$ when $p_{1}<q_{1}, a<b$; it is an increasing function of $b$ when $p_{1}<$ $q_{1}, a>b$; Further it is observed that the variability of drug efficiency is an increasing function of $b$ when $p_{1}>$ $q_{1}, a<b$; it is an increasing function of $b$ when $p_{1}>q_{1}, a$ $>b$ when other parameters are constants.

\section{Summary and Conclusions}

Our study observed that the chance of having Positive impact of the drug is giving an increasing impact on its average performance, decreasing impact on variability when $p_{1}>p_{0}$; increasing impact of variability when $p_{1}<$ $p_{0}$. The coefficient of variation is a decreasing function of performance of positive impact when $p_{1}>q_{1}$. Consistency of drug performance may be increased by maintaining more positive impact than negative impact. The chance of having Negative impact of the drug is giving an decreasing impact on its average performance, increasing impact in variability when $p_{1}>p_{0}$. The coefficient of variation is an increasing function of performance of 
Table 1. Values of mean, variance, coefficients of skewness and kurtosis for varying values of $p_{1}, q_{1}, a, b$.

\begin{tabular}{|c|c|c|c|c|c|c|c|c|}
\hline$p_{1}$ & $q_{1}$ & $\mathbf{A}$ & B & Mean & Variance & C.V. & $\beta_{1}$ & $\boldsymbol{\beta}_{2}$ \\
\hline 0.1 & 0.5 & 0.7 & 0.8 & -0.33 & 0.204 & -1.369 & 0.072 & 2.01 \\
\hline 0.2 & & & & -0.26 & 0.238 & -1.878 & 0.08 & 2.126 \\
\hline 0.3 & & & & -0.19 & 0.263 & -2.699 & 0.046 & 2.07 \\
\hline 0.4 & & & & -0.12 & 0.278 & -4.391 & 0.013 & 2.007 \\
\hline 0.6 & 0.5 & 0.7 & 0.8 & 0.02 & 0.278 & 26.344 & 0.013 & 2.007 \\
\hline 0.7 & & & & 0.09 & 0.263 & 5.697 & 0.046 & 2.07 \\
\hline 0.8 & & & & 0.16 & 0.238 & 3.052 & 0.08 & 2.126 \\
\hline 0.9 & & & & 0.23 & 0.204 & 1.964 & 0.072 & 2.01 \\
\hline 0.1 & 0.5 & 0.8 & 0.7 & -0.27 & 0.18 & -1.572 & 0.233 & 2.598 \\
\hline 0.2 & & & & -0.19 & 0.225 & -2.496 & 0.212 & 2.458 \\
\hline 0.3 & & & & -0.11 & 0.257 & -4.608 & 0.109 & 2.206 \\
\hline 0.4 & & & & -0.03 & 0.276 & -17.515 & 0.029 & 2.039 \\
\hline 0.6 & 0.5 & 0.8 & 0.7 & 0.13 & 0.276 & 4.042 & 0.029 & 2.039 \\
\hline 0.7 & & & & 0.21 & 0.257 & 2.414 & 0.109 & 2.206 \\
\hline 0.8 & & & & 0.29 & 0.225 & 1.635 & 0.212 & 0.212 \\
\hline 0.9 & & & & 0.37 & 0.18 & 1.147 & 0.233 & 2.598 \\
\hline \multirow[t]{4}{*}{0.6} & 0.2 & 0.4 & 0.5 & 0.14 & 0.078 & 2 & 0.471 & 2.625 \\
\hline & 0.3 & & & 0.09 & 0.091 & 3.35 & 0.245 & 2.26 \\
\hline & 0.4 & & & 0.04 & 0.098 & 7.842 & 0.086 & 2.039 \\
\hline & 0.5 & & & -0.01 & 0.101 & -31.765 & $9.19 \mathrm{E}-03$ & 1.967 \\
\hline \multirow[t]{4}{*}{0.6} & 0.7 & 0.4 & 0.5 & -0.11 & 0.091 & -2.741 & 0.073 & 2.26 \\
\hline & 0.75 & & & -0.135 & 0.085 & -2.163 & 0.121 & 2.427 \\
\hline & 0.8 & & & -0.16 & 0.078 & -1.75 & 0.165 & 2.625 \\
\hline & 0.85 & & & -0.185 & 0.07 & -1.433 & 0.188 & 2.832 \\
\hline \multirow[t]{4}{*}{0.6} & 0.2 & 0.6 & 0.3 & 0.3 & 0.101 & 1.058 & 0.164 & 1.658 \\
\hline & 0.3 & & & 0.27 & 0.105 & 1.202 & 0.137 & 1.726 \\
\hline & 0.4 & & & 0.24 & 0.108 & 1.369 & 0.108 & 1.753 \\
\hline & 0.5 & & & 0.21 & 0.109 & 1.571 & 0.083 & 1.761 \\
\hline \multirow[t]{4}{*}{0.6} & 0.7 & 0.6 & 0.3 & 0.15 & 0.105 & 2.163 & 0.056 & 1.726 \\
\hline & 0.75 & & & 0.135 & 0.103 & 2.38 & 0.056 & 1.699 \\
\hline & 0.8 & & & 0.12 & 0.101 & 2.646 & 0.059 & 1.658 \\
\hline & 0.85 & & & 0.105 & 0.098 & 2.98 & 0.068 & 1.597 \\
\hline \multirow[t]{4}{*}{0.6} & 0.7 & 0.1 & 0.5 & -0.29 & 0.055 & -0.808 & 0.66 & 1.864 \\
\hline & & 0.2 & & -0.23 & 0.062 & -1.083 & 0.427 & 2.071 \\
\hline & & 0.3 & & -0.17 & 0.074 & -1.601 & 0.208 & 2.223 \\
\hline & & 0.4 & & -0.11 & 0.091 & -2.741 & $7.30 \mathrm{E}-02$ & 2.26 \\
\hline \multirow[t]{4}{*}{0.6} & 0.7 & 0.6 & 0.5 & 0.01 & 0.139 & 37.269 & $6.50 \mathrm{E}-06$ & 2.114 \\
\hline & & 0.7 & & 0.07 & 0.17 & 5.892 & $7.23 \mathrm{E}-03$ & 2.006 \\
\hline & & 0.8 & & 0.13 & 0.206 & 3.492 & 0.023 & 1.901 \\
\hline & & 0.9 & & 0.19 & 0.247 & 2.615 & 0.04 & 1.807 \\
\hline \multirow[t]{4}{*}{0.7} & 0.6 & 0.1 & 0.5 & -0.23 & 0.062 & -1.083 & 0.146 & 1.287 \\
\hline & & 0.2 & & -0.16 & 0.068 & -1.635 & 0.089 & 1.571 \\
\hline & & 0.3 & & -0.09 & 0.079 & -3.121 & 0.028 & 1.869 \\
\hline & & 0.4 & & -0.02 & 0.094 & -15.297 & $4.75 \mathrm{E}-04$ & 2.087 \\
\hline \multirow[t]{4}{*}{0.7} & 0.6 & 0.6 & 0.5 & 0.12 & 0.136 & 3.069 & 0.059 & 2.256 \\
\hline & & 0.7 & & 0.19 & 0.163 & 2.124 & 0.12 & 2.257 \\
\hline & & 0.8 & & 0.26 & 0.194 & 1.696 & 0.186 & 2.234 \\
\hline & & 0.9 & & 0.33 & 0.23 & 1.454 & 0.25 & 2.199 \\
\hline \multirow[t]{4}{*}{0.6} & 0.7 & 0.4 & 0.5 & -0.11 & 0.091 & -2.741 & 0.073 & 2.26 \\
\hline & & & 0.6 & -0.18 & 0.114 & -1.876 & 0.153 & 2.247 \\
\hline & & & 0.7 & -0.25 & 0.141 & -1.504 & 0.235 & 2.208 \\
\hline & & & 0.8 & -0.32 & 0.173 & -1.299 & $3.09 \mathrm{E}-01$ & 2.16 \\
\hline \multirow[t]{4}{*}{0.6} & 0.7 & 0.4 & 0.15 & 0.135 & 0.043 & 1.538 & 0.097 & 1.532 \\
\hline & & & 0.2 & 0.1 & 0.047 & 2.163 & 0.056 & 1.726 \\
\hline & & & 0.25 & 0.065 & 0.052 & 3.492 & 0.023 & 1.901 \\
\hline & & & 0.3 & 0.03 & 0.057 & 7.979 & $3.44 \mathrm{E}-03$ & 2.042 \\
\hline \multirow[t]{4}{*}{0.7} & 0.6 & 0.4 & 0.5 & -0.02 & 0.094 & -15.297 & $4.75 \mathrm{E}-04$ & 2.042 \\
\hline & & & 0.6 & -0.08 & 0.12 & -4.33 & 0.014 & 1.953 \\
\hline & & & 0.7 & -0.14 & 0.151 & -2.777 & 0.036 & 1.83 \\
\hline & & & 0.8 & -0.2 & 0.187 & -2.163 & 0.056 & 1.726 \\
\hline \multirow[t]{4}{*}{0.7} & 0.6 & 0.4 & 0.15 & 0.19 & 0.039 & 1.039 & 0.458 & 2.046 \\
\hline & & & 0.2 & 0.16 & 0.043 & 1.299 & 0.309 & 2.16 \\
\hline & & & 0.25 & 0.13 & 0.049 & 1.696 & 0.186 & 2.234 \\
\hline & & & 0.3 & 0.1 & 0.055 & 2.349 & 0.099 & 2.261 \\
\hline
\end{tabular}



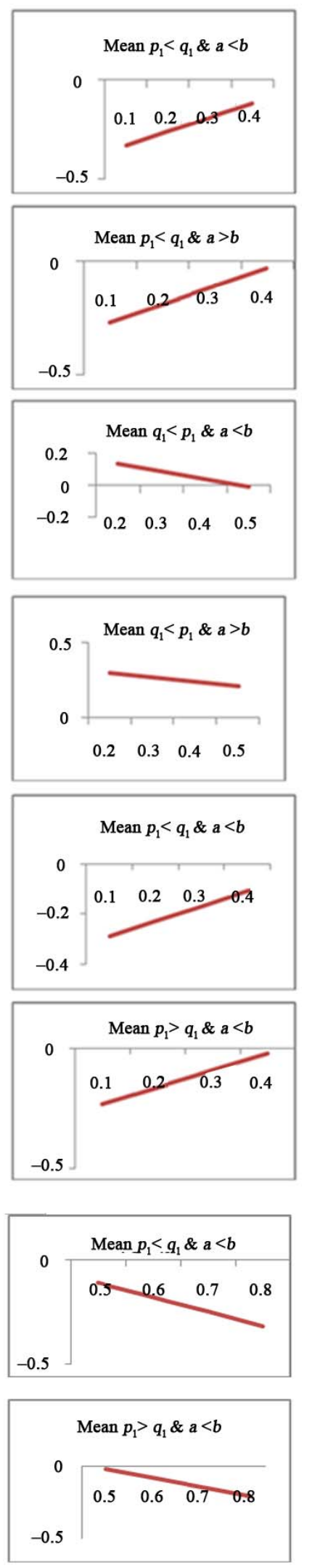
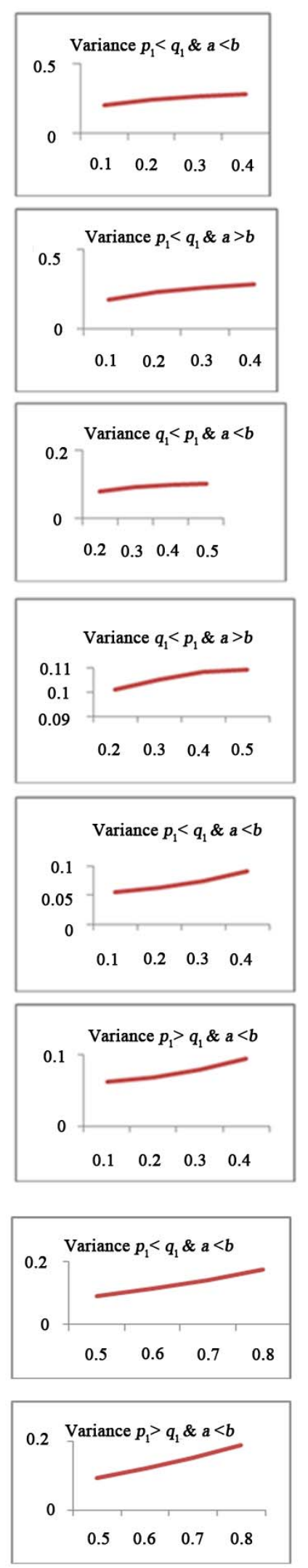
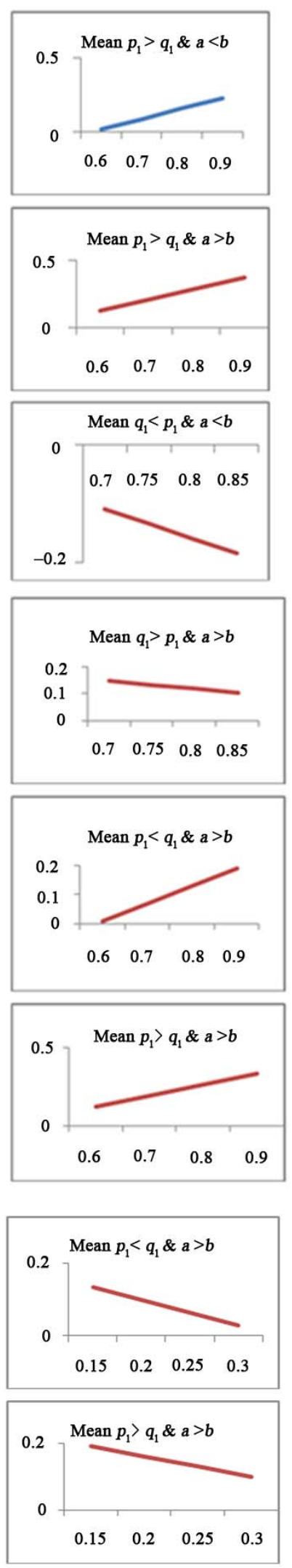
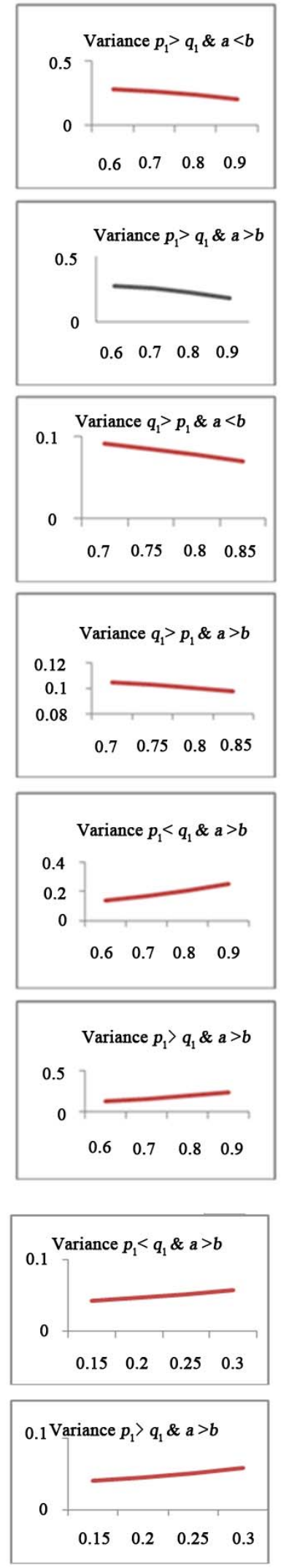

Figure 1. Graphical presentations of mean and variance effects of drug. 
Negative impact when $p_{1}>q_{1}$. The chance of having Positive impact of the drug is giving an increasing impact on its average performance, increasing impact on variability when $a>b$. The coefficient of variation is a decreasing function of performance of positive impact of drug when $a>b$. Consistency of drugs performance may be increased by maintaining more positive impact than negative impact. This model will help the individual patients in quantification of the problem severity with drug abuse/misuse. Development of software to this model will assist in the health monitoring of self health care takers fort their decision support systems. The scope for future work may be done with multinomial cases instead of Bernoulli cases. This work may be extended to more suitable contexts.

\section{Acknowledgements}

All the authors of the paper are very much greateful to the editorial board, reviewers of the Paper, and Editors of the Journal of Service Science and Management (JSSM) for their valuable suggestion in improving the quality of the paper.

\section{REFERENCES}

[1] S. H. Daukes, "A Consideration of the Various Methods of Drug Administration," Post Graduate Medical Journal, Vol. 5, No. 49, 1929, pp. 1-7. doi:10.1136/pgmj.5.49.1

[2] J. Smieja, et al., "Optimal Control for the Model of Drug Resistance Resulting from Gen Amplification," Preperation of the 14th IFAC World Congress, Beijing, China, Vol. 50, 1999, pp. 71-75.

[3] A. Swierniak et al., "Asymptotic Properties of Infinite Dimensional Model Drug Resistance Evolution," Proceedings of the European Control Conference (ECC), Brussels, 1997.

[4] W. E. Evans and H. L. McLeod, "Pharmacogenomicsdrug disposition, drug targets, and side effects," New England Journal of Medicine, Vol. 348, No. 6, 2003, pp. 538-549. doi:10.1056/NEJMra020526

[5] C. Abad-Zapatero and J. T. Metz, "Ligand Efficiency Indices as Guideposts for Drug Discovery," Drug Discovery Today, Vol. 10, No. 7, 2005, pp. 464-469. doi:10.1016/S1359-6446(05)03386-6

[6] C. Hetenyi, et al., "Structure-Based Calculation of Drug Efficiency Indices," Journal of Structural Bioinformatics, Vol. 23, No. 20, 2007, pp. 2678-2685.

doi:10.1093/bioinformatics/btm431

[7] P. Tirupathi Rao and R. Bharathi, "Stochastic Modeling in the Evaluation of Drug Efficiency," International Journal of Statistics and System, Vol. 5, No. 3, 2010, pp. 427-438. 